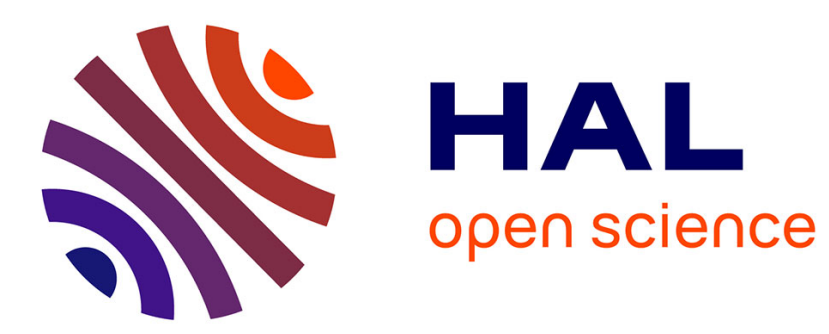

\title{
Fluorocarbon Exposure Mode Markedly Affects Phospholipid Monolayer Behavior at the Gas/Liquid Interface. Impact on Size and Stability of Microbubbles
}

Da Shi, Xianhe Liu, Claire Counil, Marie Pierre Krafft

\section{- To cite this version:}

Da Shi, Xianhe Liu, Claire Counil, Marie Pierre Krafft. Fluorocarbon Exposure Mode Markedly Affects Phospholipid Monolayer Behavior at the Gas/Liquid Interface. Impact on Size and Stability of Microbubbles. Langmuir, 2018. hal-03018421

\section{HAL Id: hal-03018421 https://hal.science/hal-03018421}

Submitted on 22 Nov 2020

HAL is a multi-disciplinary open access archive for the deposit and dissemination of scientific research documents, whether they are published or not. The documents may come from teaching and research institutions in France or abroad, or from public or private research centers.
L'archive ouverte pluridisciplinaire HAL, est destinée au dépôt et à la diffusion de documents scientifiques de niveau recherche, publiés ou non, émanant des établissements d'enseignement et de recherche français ou étrangers, des laboratoires publics ou privés. 
Fluorocarbon Exposure Mode Markedly Affects Phospholipid Monolayer Behavior at the Gas/Liquid Interface. Impact on Size and Stability of Microbubbles

Da Shi, Xianhe Liu, Claire Counil, Marie Pierre Krafft*

University of Strasbourg, Institut Charles Sadron (CNRS). 23 rue du Loess. 67034 Strasbourg Cedex (France)

${ }^{*}$ Corresponding author

krafft@unistra.fr 


\begin{abstract}
Although most phospholipid-shelled microbubbles (MBs) investigated for medical applications are stabilized by a fluorocarbon $(F C)$ gas, information on the interactions between phospholipid and $F C$ molecules at the gas/water interface remains scarce. We report that the procedure of introduction of perfluorohexane ( $F$-hexane), that is, either in the gas phase above dimyristoylphosphatidylcholine (DMPC) or dipalmitoylphosphatidylcholine (DPPC) Langmuir monolayers, or in the aqueous sub-phase, radically affects the compression isotherms. When introduced in the gas phase, $F$-hexane is rapidly incorporated in the interfacial film but is also readily desorbed upon compression and eventually totally expelled from the phospholipid monolayers. By contrast, when introduced in the aqueous phase, F-hexane remains trapped at the interface. These dissimilar outcomes demonstrate that the phospholipid monolayer acts as a barrier that effectively hinders the transfer of the FC across the interfacial film. F-hexane was also found to significantly accelerate the adsorption kinetics of the phospholipids at the gas/water interface and to lower the interfacial tension, as assessed by bubble profile analysis tensiometry. The extent of these effects is more pronounced when $F$-hexane is provided from the gas phase. The size and stability characteristics of DMPC- and DPPC-shelled microbubbles were also found to depend on how the $F C$ is introduced. As compared to reference MBs prepared under nitrogen only, introduction of $F$-hexane always causes a decrease in MB mean radius. However, whilst for DMPC this decrease depends on F-hexane introduction procedure, it is independent from procedure and most pronounced (from $\sim 2.0 \mu \mathrm{m}$ to $\sim 1.0 \mu \mathrm{m}$ ) for DPPC. Introducing the FC in the gas phase has the strongest effect on MB half-life $\left(t_{1 / 2}=\sim 1.8\right.$ and $6.8 \mathrm{~h}$ for DMPC and DPPC, respectively), as compared to when it is delivered through the aqueous phase $(\sim 0.8$ and $\sim 1.7 \mathrm{~h}$ ). Fluorocarbon-less reference DMPC and DPPC bubbles had a half-life of $\sim 0.5$ and $0.8 \mathrm{~h}$, respectively. The effects of $F$-hexane on MB characteristics are discussed with regards to the interactions between phospholipids and $F$-hexane and monolayer fluidization effect as revealed by the Langmuir and tensiometric studies.
\end{abstract}




\section{Introduction}

Micrometer-size gas bubbles are being used and investigated as contrast agents for ultrasound diagnostic imaging, molecular imaging, targeted drug and gene delivery systems, and as mechanical intravascular devices. ${ }^{1-10}$ When subjected to an ultrasound field, microbubbles (MBs) alternatively shrink and expand in response to acoustic pressure. The volume expansion of the MBs is maximal at a specific frequency, which is inversely related to their size. The backscatter echo produced by an MB at resonance provides valuable images of tissues and organs in their neighborhood. More powerful sound pulses can be used to trigger bubble rupture and release of a drug cargo, which enables the use of MBs as theranostic agents.

Fluorocarbon $(F C)$ gases are commonly used to stabilize phospholipid-shelled air microbubbles destined to medical applications. The MB-based contrast agents that are commercially available contain $\mathrm{SF}_{6}, \mathrm{C}_{3} \mathrm{~F}_{8}$ or $\mathrm{C}_{4} \mathrm{~F}_{10} .^{11-12}$ Other $\mathrm{FCs}$, such as $\mathrm{C}_{5} \mathrm{~F}_{12}$ and $\mathrm{C}_{6} \mathrm{~F}_{14}$ have also been extensively investigated. ${ }^{1}$ Further examples of potential medical applications of FC gases include injectable oxygen carriers, ${ }^{13}$ lung surfactant replacement compositions, ${ }^{14-17}$

The stabilizing role of FCs on MBs was initially assigned to an osmotic effect, the poorly soluble hydrophobic FC gas having a substantially lesser solubility and propensity to diffuse into the continuous aqueous phase than $\mathrm{O}_{2}$ and $\mathrm{N}_{2} \cdot{ }^{18}$ The role of the $\mathrm{FC}$ in the bubble stabilization mechanism was solely deemed to osmotically counteract the blood pressure and Laplace pressure that operate on and tend to shrink the bubbles, thus opposing their dissolution in the blood. Intravascular bubble stability was foretold to increase with decreasing Ostwald coefficient of the gas. A lower limit was set for the FC's vapor pressure $\left(\sim 0.310^{5} \mathrm{~Pa}\right)$ below which condensation of the $\mathrm{FC}$ into a liquid would occur under the action of the combined external pressures, resulting in bubble collapse. The interfacial film, usually a monolayer of phospholipids, and its possible interactions with the fluorocarbon, were not taken into consideration. While the predicted general trend was confirmed experimentally both in vitro and in vivo, the experimentally measured bubble half-life values were always 
larger than the predicted ones by several orders of magnitude. ${ }^{19}$ Convincingly, the fact that small-sized bubbles can be stabilized by F-triglyme, for which no osmotic stabilization can be expected due to its very low vapor pressure $\left(0.0110^{5} \mathrm{~Pa}\right.$ at $\left.25^{\circ} \mathrm{C}\right)$, implied that the osmotic mechanism is not the only one to at work. ${ }^{20}$ We subsequently established that the $F C$ gas also interacts with the shell of phospholipids, and actually acts as an effective cosurfactant. $^{21}$

Although there are some reports about the interactions between highly amphiphilic fluorinated surfactants and phospholipid membranes, ${ }^{22-25}$ the studies that seek a better understanding of the interactions of fluorocarbons, which are non polar and non amphiphilic, with phospholipid monolayers very limited.$^{26}$ In practice, gas MBs are generally prepared by injecting a buffer solution in sealed vials that contain both the lyophilized phospholipid shell components and the FC gas, and submitting this mixture to mechanical agitation. ${ }^{27}$ One should remember that, although the solubility of FCs in water is very low, it is not null. The concentration of $F$-hexane, $F$-pentane, $F$-butane, and $F$-propane in water are estimated to $2.710^{-4}, 4.010^{-3}, 0.021$, and $0.19 \mathrm{~mol} \mathrm{~m}^{-3}$, respectively. ${ }^{19}$ It is therefore legitimate to wonder about the capacity for FCs to cross the interfacial phospholipid film and diffuse into the aqueous phase. In particular, the impact of introducing the $F C$ in the aqueous phase in which the MBs are dispersed, rather than in the gas phase on their phospholipid shell was unknown.

We first present here a basic study of the impact of introducing a fluorocarbon, $F$-hexane, in the aqueous sub-phase on the adsorption and behavior of phospholipids (dimyristoylphosphatidylcholine DMPC and dipalmitoylphosphatidylcholine DPPC) at the air/water interface. We therefore compared the effects on compression isotherms and adsorption rates depending on whether the fluorocarbon is introduced in the gas phase, in the aqueous phase, or in both, using both Langmuir film compression experiments and bubble shape analysis tensiometry. In a second part, we test the consequences of the FC introduction mode on the size and stability characteristics of DMPC- or DPPC-shelled MBs. 


\section{Experimental part}

Materials. 1,2-Dimyristoylphosphatidylcholine $\quad$ (DMPC) and 1,2dipalmitoylphosphatidylcholine (DPPC) were purchased as dry powders (99\% purity) from Avanti Polar Lipids (Alabaster, AL) and used as received. Perfluorohexane (98\% pure) was from Fluorochem. Pluronic F-68 (a poly(ethylene oxide)-poly(propylene oxide) triblock copolymer, $M W \approx 8300$, purity $>99 \%$ ) and HEPES (N-(2-hydroxyethyl)piperazine-N'-2ethanesulfonic acid) were from Sigma-Aldrich (Lyon, France). A HEPES buffer solution (20 $\mathrm{mmol} \mathrm{L}^{-1}$ ) in a $150 \mathrm{mmol} \mathrm{L}^{-1} \mathrm{NaCl}$ solution was prepared and its $\mathrm{pH}$ was adjusted to 7.4 with $1 \mathrm{~N} \mathrm{NaOH}$. Water was purified using a Millipore system (surface tension $71.4 \mathrm{mN} \mathrm{m}^{-1}$ at $20^{\circ} \mathrm{C}$, resistivity $\left.18.2 \mathrm{M} \Omega \mathrm{cm}\right)$.

Langmuir monolayers. The surface pressure $(\pi)$ versus molecular area $(A)$ isotherms were recorded using a Langmuir minitrough (Riegler \& Kirstein, Potsdam, Germany) equipped with two movable barriers (initial area: $204 \times 60 \mathrm{~mm}$, compression speed: $36.5 \mathrm{~mm}^{2} \mathrm{~s}^{-1}$, which corresponds to a reduction of the total area of $\left.\sim 18 \% \min ^{-1}\right)$. $\pi$ was measured using the Wilhelmy plate (paper) method. The trough was enclosed in a Plexiglas box (volume $\sim 9 \mathrm{~L}$ ) and maintained at $25 \pm 0.5{ }^{\circ} \mathrm{C}^{15} 15 \mu \mathrm{L}$ of a DMPC or DPPC solution $\left(1 \mathrm{mmol} \mathrm{L}{ }^{-1}\right)$ in chloroform were spread on the surface of the HEPES buffer solution $(75 \mathrm{~mL})$ and $15 \mathrm{~min}$ were allowed for chloroform evaporation. For the experiments in which F-hexane was introduced in the gas phase, saturation of air was achieved by depositing $F$-hexane (a liquid at room temperature; $20 \mathrm{~mL}$ ) in small aluminum dishes within the air-containing box. For the experiments in which $F$-hexane was introduced in the aqueous sub-phase, saturation of the HEPES solution with F-hexane was achieved by depositing $200 \mu \mathrm{L}$ of $F$-hexane at the bottom of the HEPES solution $(100 \mathrm{~mL})$. The mixture was stirred for $3-6 \mathrm{~h}$ at room temperature and allowed to rest for $6 \mathrm{~h}$ before compression was initiated.

Bubble profile analysis tensiometry. Axisymmetric bubble shape analysis was applied to a rising bubble of gas (air or F-hexane-saturated air) formed in a dispersion of DMPC or DPPC vesicles in an aqueous phase (HEPES buffer or F-hexane-saturated HEPES buffer). The 
vesicles were prepared by sonication of DMPC and DPPC dispersions $\left(10^{-3} \mathrm{~mol} \mathrm{~L}^{-1}\right)$ in HEPES buffer $(50 \mathrm{~mL})$ for $30 \mathrm{~min}$ at $30^{\circ} \mathrm{C}$ and $50^{\circ} \mathrm{C}$, respectively. Care was taken to only use vesicle dispersions having mean diameters of $70-80 \mathrm{~nm}$ and narrowly dispersed size distributions (Supporting information, Fig. S1). The time dependence of the interfacial tension during adsorption of the phospholipid at the gas/liquid interface was measured using a Tracker ${ }^{\circledR}$ tensiometer (Teclis, Civrieux d'Azergues, France). The bubble $(5 \mu \mathrm{L})$ was formed at the end of a steel capillary with a tip diameter of $1 \mathrm{~mm}$. Saturation of the rising bubble with F-hexane was achieved by purging a $1 \mathrm{~mL}$ syringe three times with $F$-hexane-saturated air (taken above liquid $F$-hexane) according to ${ }^{21,28}$. This syringe was immediately mounted on the injection cell of the tensiometer and the rising bubble formed. Saturation of the aqueous phase with $F$-hexane was achieved by depositing $500 \mu \mathrm{L}$ of the fluorocarbon at the bottom of the measuring glass cell $(10 \mathrm{~mL}$, closed by a lid) that contains the vesicle dispersions. $90 \mathrm{~min}$ were allowed before forming the rising bubble. F-hexane-saturated vapor pressure and concentration at $25^{\circ} \mathrm{C}$ are $2.910^{4} \mathrm{~Pa}$ and $11.66 \mathrm{~mol} \mathrm{~m}^{-3}$, respectively, and water solubility at $25^{\circ} \mathrm{C}$ is $2.710^{-4} \mathrm{~mol} \mathrm{~m}^{-3}$. The characteristic adsorption time $\tau$ was calculated by fitting the adsorption profiles with an exponential decay function.

Preparation of the microbubble dispersions. Four series of $\mathrm{MB}$ dispersions were prepared for each phospholipid in a non-degassed buffer solution. The first one was obtained by dispersing DMPC or DPPC and Pluronic F-68 (10:1 molar ratio) in the HEPES buffer under nitrogen, and constitutes the reference MB dispersions. In the second series, the buffered aqueous phase was saturated with $F$-hexane droplets deposited at the bottom of the vial. In the third series, the gas phase $\left(\mathrm{N}_{2}\right)$ from which the bubbles were prepared was saturated by vapors of $F$-hexane (see protocol below). In the fourth series, $F$-hexane was introduced in both the aqueous and gaseous phases.

\section{Microbubbles having a gas phase of $\mathrm{N}_{2}$ (series 1) or of $\mathrm{N}_{2}$ saturated with F-hexane} (series 2). DMPC or DPPC (50 mmol L-1) and Pluronic F-68 (DMPC/F-68 molar ratio 10:1) were dispersed in a HEPES buffer $(1 \mathrm{~mL})$ in a glass tube (inner diam. $18 \mathrm{~mm}$, length $63 \mathrm{~mm}$ ) 
by magnetic stirring for $3-6 \mathrm{~h}$ at room temperature. Pluronic F-68 was added to facilitate phospholipid dispersion and foam formation. The dispersions were pre-sonicated under air at low power (setting 2) for $30 \mathrm{~s}$ at $25^{\circ} \mathrm{C}$. The sonicator (Vibracell, Bioblock Scientific, Illkirch, France) was equipped with a $3 \mathrm{~mm}$ titanium probe and operated at $20 \mathrm{kHz}$ with an output power of $\sim 600 \mathrm{~W}$ (duty cycle $40 \%$ ). The resulting dispersions were sonicated for $15 \mathrm{~s}$ (setting 5 , duty cycle $40 \%$ ) at $25^{\circ} \mathrm{C}$ under $\mathrm{N}_{2}$ (series 1 ) or under $F$-hexane-saturated $\mathrm{N}_{2}$ (series 2) at $30^{\circ} \mathrm{C}$ for DMPC and $50^{\circ} \mathrm{C}$ for DPPC (i.e. above the transition phase temperatures of the phospholipids). For series $2, \mathrm{~N}_{2}$ was allowed to bubble through three vials containing $F$-hexane before being flushed into the glass tube. The probe was consistently positioned $5 \mathrm{~mm}$ below the surface of the dispersion. The resulting foam was immediately diluted with $14 \mathrm{~mL}$ of HEPES buffer. Size fractionation of the microbubbles was achieved by flotation for 30 and 60 min for DMPC and DPPC, respectively. ${ }^{29}$

Microbubbles dispersed in an aqueous phase saturated with F-hexane (series 3). Saturation of the HEPES solution with $F$-hexane was achieved by depositing $200 \mu \mathrm{L}$ of $F$ hexane at the bottom of a vial containing the HEPES solution $(100 \mathrm{~mL})$, stirring $(3-6 \mathrm{~h})$ at room temperature and allowing the resulting dispersion to equilibrate for $6 \mathrm{~h}$. DMPC or DPPC $\left(50 \mathrm{mmol} \mathrm{L}^{-1}\right)$ and Pluronic F-68 $\left(5 \mathrm{mmol} \mathrm{L}^{-1}\right)$ were dispersed in aliquots $(1 \mathrm{~mL})$ sampled from the supernatant of the F-hexane-saturated buffer by stirring for one night at room temperature in a closed glass tube (inner diam. $18 \mathrm{~mm}$, length $63 \mathrm{~mm}$ ). Pre-sonication and sonication of the dispersions were achieved as described above. The resulting foam was diluted with $14 \mathrm{~mL}$ of the F-hexane-saturated HEPES buffer and size fractionation was achieved as above.

Microbubbles with F-hexane introduced in both the gaseous and aqueous phases (series 4). Preparation and pre-sonication of $1 \mathrm{~mL}$ of the DMPC or DPPC/Pluronic F-68 (molar ratio 10:1) dispersions in F-hexane-saturated HEPES buffer were achieved by applying the same procedure as for series 3 . Sonication involved saturation of the gas phase with $F$-hexane as for series 2 . The resulting foam was diluted with $14 \mathrm{~mL}$ of the $F$-hexanesaturated HEPES buffer. Size fractionation was achieved as above. 


\section{Characterization of microbubble dispersions}

Optical microscopy. Three to four droplets of bubble dispersion were placed into a concave glass slide and covered with a glass slide. The samples were observed with a Nikon Eclipse 90i microscope (transmission mode). Rapid image acquisition was achieved using a Lumenera Infinity 2 charge-coupled device (CCD) camera (Lumenera, Ottawa, Canada). Bubble radii were measured using the ImageJ software on 5-10 slides.

Acoustic size determination. The method exploits the sound attenuation undergone by multi-frequencies ultrasound waves that propagate through the aqueous bubble dispersion. Standard simple-harmonic resonator curves are fitted to measured attenuations in order to infer the size of the bubbles. A low-power emitter is used so as to avoid alteration of the bubble characteristics and stability. For experimental details see ${ }^{30}$. Each measurement was repeated three times on different bubble preparations. The volume of microbubble dispersion injected in the acoustic cell was $2 \mathrm{~mL}$.

\section{Results}

Comparing compression isotherms of Langmuir monolayers of phospholipids while Fhexane is introduced above or beneath the monolayer, or both

The compression isotherms of DMPC and DPPC monolayers were investigated while $F$ hexane was introduced in the gas phase above the monolayer, or in the aqueous sub-phase, or in both, and compared to the isotherms of the phospholipid measured in the absence of fluorocarbon (Fig. 1). For both phospholipids, the introduction of $F$-hexane always leads to an increase of the surface pressure $\pi$ at large molecular areas $A$, reflecting the insertion of the FC into the phospholipid monolayers. However, one observes that, when introduced in the gas phase, $F$-hexane is progressively desorbed upon compression and eventually totally expelled, since the isotherm rejoins that measured in the absence of $F C\left(A=45 \pm 2 \AA^{2}\right.$ at $\pi$ $=46 \mathrm{mN} \mathrm{m}^{-1}$ ). On the contrary, the $F C$ remains trapped at the interface when introduced in the aqueous sub-phase, as evidenced by the larger molecular area $\left(A=60 \pm 2 \AA^{2}\right.$ for DMPC and $52 \pm 2 \AA^{2}$ for DPPC at $\pi=46 \mathrm{mN} \mathrm{m}^{-1}$ ). In addition, $F$-hexane has a strong influence on 
the liquid-expanded (LE) to liquid-condensed (LC) phase transition of the DPPC monolayer. When the $F C$ is delivered from the gas phase, the LE/LC transition is shifted towards larger pressures but occurs at the same molecular area $\left(\sim 80 \AA^{2}\right)$. By contrast, when the $F C$ is provided via the aqueous phase, the transition is shifted towards larger areas, while the pressure remains unchanged $\left(\sim 10 \mathrm{mN} \mathrm{m}^{-1}\right)$. This behavior strongly supports the view that the interaction of $F$-hexane with the phospholipid is relatively weak when the former comes from the gas phase; the FC is indeed readily expelled towards the gas phase. By contrast, when the $F C$ is provided from the aqueous phase, its interaction with the phospholipid interface is stronger, which enables the FC to be trapped at the interface. When present in both gaseous and aqueous phases, the FC causes as well an increase of surface pressure $\pi$ at large molecular area, as the immobilization of the $F C$ at small molecular area and the inhibition of the LE/LC transition.
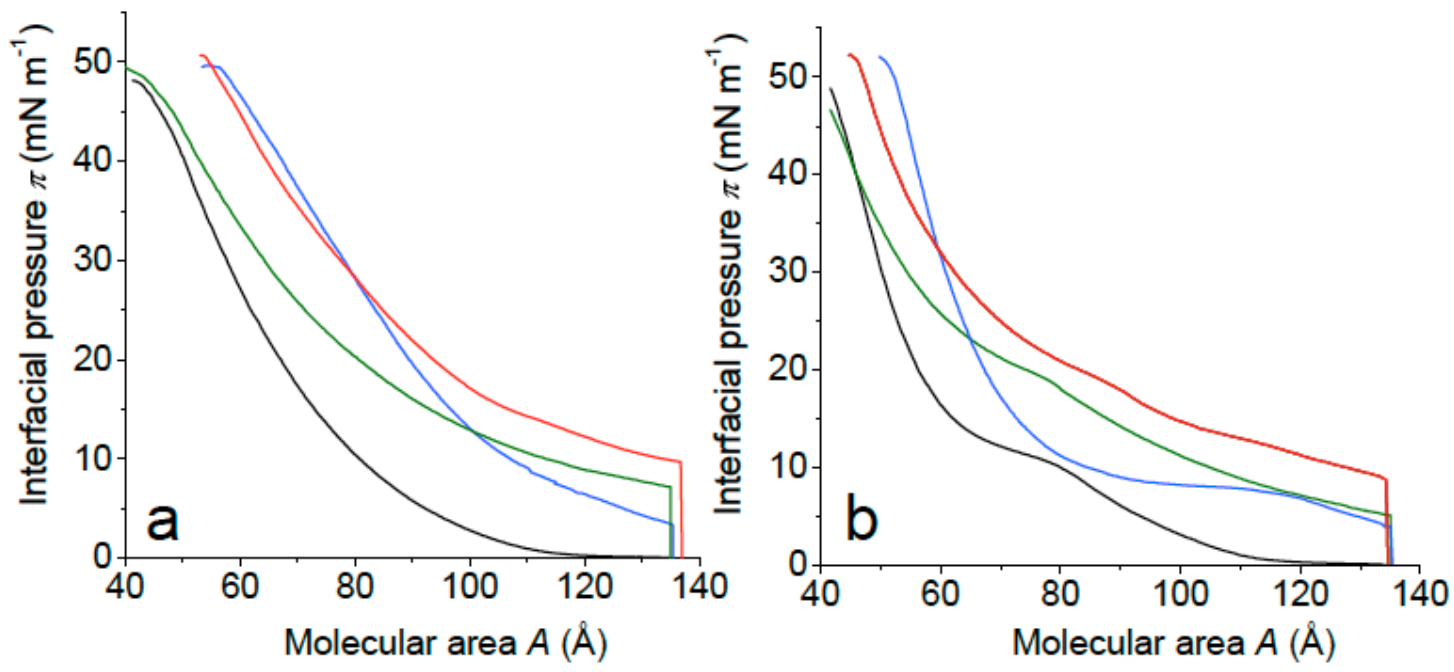

Figure 1. Compression isotherms of a) DMPC and b) DPPC monolayers $\left(25^{\circ} \mathrm{C}\right)$. The monolayers were spread on a buffered aqueous sub-phase under air (black) and under air saturated with $F$-hexane (green); or they were spread under air on a sub-phase saturated with $F$-hexane (blue); finally $F$-hexane was introduced both in the air and aqueous phases (red). 


\section{Changes in adsorption kinetics of phospholipids when F-hexane is introduced above or beneath the monolayer, or both}

First, we measured the effect of $F$-hexane on the interfacial tension $\sigma$ between the aqueous and gas phases, the fluorocarbon being introduced in either one or the other phase, using bubble shape analysis tensiometry. Figure 2 shows that $F$-hexane decreases significantly $\sigma$ (from $\sim 72$ to $67 \mathrm{mN} \mathrm{m}^{-1}$ ) at equilibrium, both when provided via the gaseous or the aqueous phase. The FC adsorption kinetics are however markedly different. When in the gas phase $F$-hexane adsorbs rapidly at the interface (the adsorption is actually too fast to be monitored by our tensiometer), as shown in an earlier report. ${ }^{28}$ On the other hand, when provided via the aqueous phase, the FC adsorption is strongly slowed down (adsorption characteristic time $\tau \sim 1.8 \mathrm{~h}$ ).

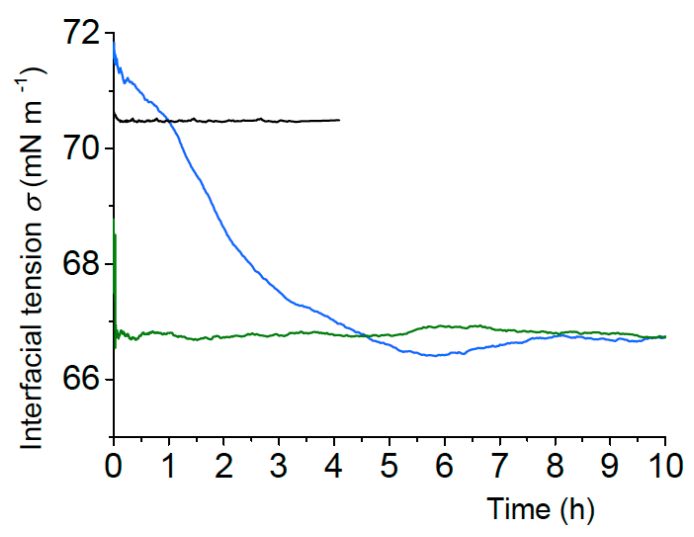

Figure 2. Variation of the interfacial tension $\sigma$ of the gas/aqueous phase interface as a function of time under air (black); when $F$-hexane is provided from the gas phase (green); and from the aqueous phase (blue). Temperature was $25^{\circ} \mathrm{C}$.

Next, we investigated spontaneously adsorbed (Gibbs) films of DMPC and DPPC at the gas/buffer interface in the presence of $F$-hexane, the latter being introduced either in the gaseous or in the aqueous phase (Fig. 3). The two phospholipids were provided in the form of similarly sized vesicles. 

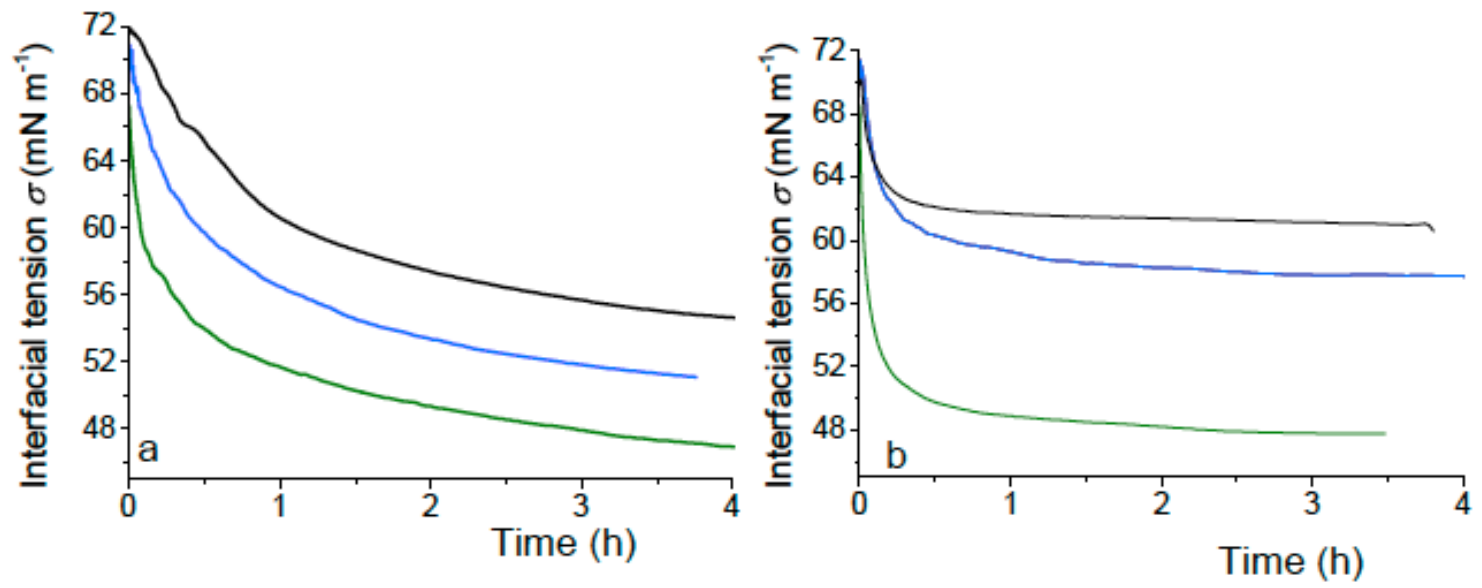

Figure 3. Adsorption kinetics of a) DMPC and b) DPPC at the gas/buffer interface $\left(25^{\circ} \mathrm{C}\right)$; Air only (black); $F$-hexane introduced in the gas phase (green); and $F$-hexane deposited in the aqueous phase (blue).

F-hexane is observed to strongly accelerate the adsorption of both phospholipids and decrease their interfacial tension at equilibrium $\left(\sigma_{\mathrm{eq}}\right)($ Table 1$)$.

Table 1. Characteristic adsorption times $\tau$ for DMPC and DPPC vesicles depending on $F$ hexane introduction mode.

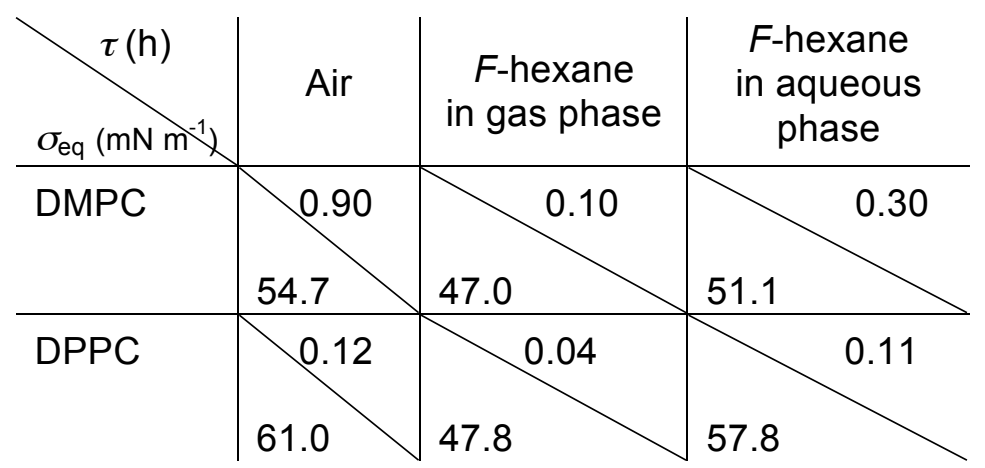

The extent of these effects depends however on the phospholipid and on how the FC is introduced. DPPC adsorbs faster than DMPC, which likely reflects more numerous topological defects present in the gel-phase bilayer of DPPC vesicles. ${ }^{31}$ The adsorption of phospholipid vesicles at the air/water interface has indeed been described to occur via a first slow irreversible transformation of the water-dispersible vesicles into adsorbed vesicles containing bilayer defects, followed by a fast spreading of the phospholipid molecules at the interface. ${ }^{32}$ For both phospholipids, the adsorption was the fastest and $\sigma_{\text {eq }}$ values the lowest 
when the FC was introduced in the gas phase. These results are in line with the compression isotherm study that indicated that the phospholipids acted as a barrier that opposes the transfer of the $F C$ from the aqueous phase to the gaseous phase.

\section{Preparation and characterization of fluorocarbon gas-stabilized microbubbles}

The size and stability characteristics of a series of microbubbles were investigated by optical microscopy and by ultrasound wave attenuation analysis. DMPC was selected as the bubble's shell component because this phospholipid does normally not yield very stable MBs, and therefore facilitates the assessment of any stabilization or destabilization effect. DPPC was selected because it is the prominent component of most practical MB formulations. A series of experiments was achieved that aimed at evaluating the impact of $F$-hexane on MB characteristics, depending on whether the FC is provided through the gas phase, by saturating $\mathrm{N}_{2}$ with vapors of $F$-hexane (Scheme $1 \mathrm{~b}$ ), or through the continuous aqueous phase by saturating it with the FC (Scheme 1c), or both (Scheme 1d). MBs prepared without fluorocarbon were prepared as a reference.
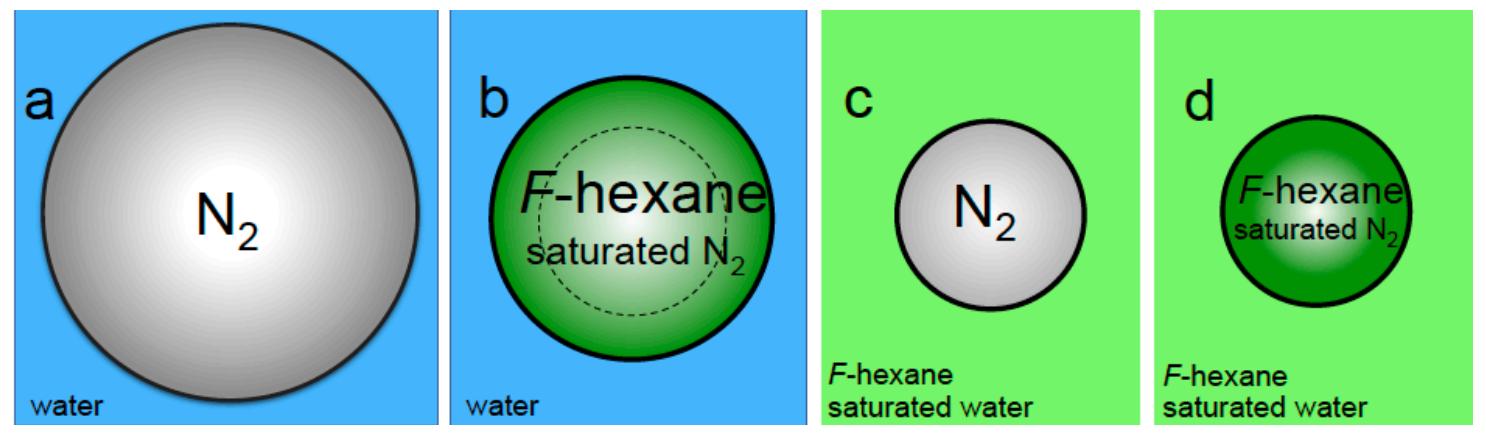

Scheme 1. Experiments designed for assessing the effect of the mode of introduction of $F$ hexane on the size and stability characteristics of DMPC- or DPPC-shelled MBs. $\mathrm{N}_{2}$ MBs (a and $c$ ), or $F$-hexane-saturated $\mathrm{N}_{2} \mathrm{MBs}$ ( $\mathrm{b}$ and $\mathrm{d}$ ) prepared in the aqueous buffer solution (a and $b$ ), or in an F-hexane-saturated aqueous solution (c and $d$ ). The cartoon reflects the relative size of the bubbles. In b), the solid and dotted lines correspond to DMPC and DPPC microbubbles, respectively. 
The stability of the MBs was monitored by the acoustical attenuation method. The variation of the attenuation coefficient, $\alpha$, of the ultrasound wave as a function of the ultrasound frequency, $f$, was determined at the initial measuring time, $t_{0}$ (i.e. $32 \mathrm{~s}$ after injection of the microbubbles in the acoustical measuring cell. The evolution of the radius distributions with time was determined from the attenuation curves (see an example in Fig. 4 for DMPC; for DPPC microbubbles prepared in other conditions see Supporting Information, Fig. S2-5). In all cases, the mean bubble radius remained essentially constant over time, while their number decreased. The bubble fraction was then plotted as a function of time, allowing determination of the microbubbles' half-life. ${ }^{30}$

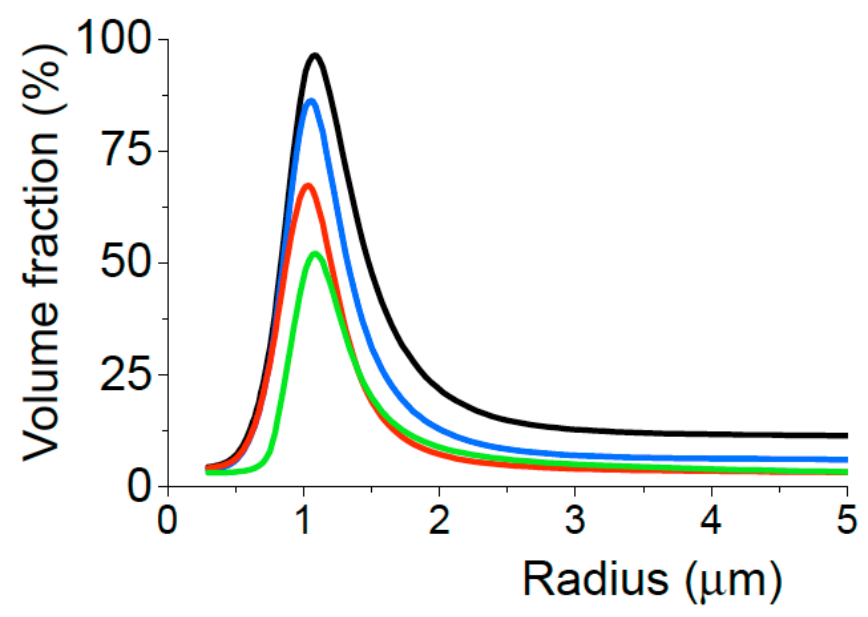

Figure 4. Time evolution at $25^{\circ} \mathrm{C}$ of the size distributions of DMPC-shelled microbubbles when $F$-hexane was introduced in the gas phase (black: immediately after preparation; blue: after $0.44 \mathrm{~h}$; red: $1 \mathrm{~h}$ and green: $3 \mathrm{~h}$ ).

The size and stability characteristics of the various DMPC- and DPPC-shelled MBs prepared are provided in Table 2 and Figures 5-6. For DMPC, the mean radius of reference MBs prepared without addition of $F$-hexane is $\sim 2.0 \mu \mathrm{m}$, as assessed by both optical microscopy and the acoustical attenuation method. Their half-life is $\sim 0.5 \mathrm{~h}$. These characteristics are in agreement with a previous report. ${ }^{20}$ 
Table 2. Physical characteristics of DMPC- and DPPC-shelled microbubbles depending on $F$-hexane exposure mode

$\begin{array}{ll}\mathrm{N}_{2} & \begin{array}{l}F \text {-hexane } \\ \text { in gas phase }\end{array}\end{array}$

$\begin{array}{ll}\text { F-hexane } & F \text {-hexane } \\ \text { in aqueous } & \text { in both aqueous } \\ \text { phase } & \text { and gas phases }\end{array}$

DMPC

$R_{\text {mean }}$ (optical, $\left.\mu \mathrm{m}\right)$

$2.0 \pm 0.3 \quad 1.5 \pm 0.3$

$1.0 \pm 0.2$

$1.0 \pm 0.1$

$R_{\text {mean }}$ (acoustical, $\mu \mathrm{m}$ )

$2.4 \pm 0.1 \quad 1.8 \pm 0.2$

$1.1 \pm 0.1$

$1.0 \pm 0.2$

Half-life $\left(t_{1 / 2}, h\right)$

$0.5 \pm 0.1 \quad 1.8 \pm 0.1$

$0.8 \pm 0.1$

$1.8 \pm 0.2$

DPPC

$R_{\text {mean }}$ (optical, $\mu \mathrm{m}$ )

$R_{\text {mean }}$ (acoustical, $\mu \mathrm{m}$ )

Half-life $\left(t_{1 / 2}, h\right)$

$2.0 \pm 0.2$
$1.6 \pm 0.1$
$0.8 \pm 0.2$

$0.9 \pm 0.1$

$0.8 \pm 0.2$

$1.0 \pm 0.2$

$0.8 \pm 0.1$

$0.8 \pm 0.2$

$6.8 \pm 0.5$

$1.0 \pm 0.1$

$0.9 \pm 0.2$

$1.7 \pm 0.2$

$7.2 \pm 0.5$

When $F$-hexane is introduced in the gas phase, the mean radius of DMPC-shelled MBs decreases (to $\sim 1.5 \mu \mathrm{m}$ ) and the half-life $(1.8 \mathrm{~h}$ ) increases as compared to the reference MBs. Introduction of $F$-hexane in the aqueous phase leads to a further decrease in mean radius (to $\sim 1.0 \mu \mathrm{m}$ ) with a half-life of $0.8 \mathrm{~h}$, which is significantly longer than for reference MBs. Finally, when $F$-hexane is introduced in both gaseous and aqueous phases, one observes both a decrease in mean radius (to $\sim 1.0 \mu \mathrm{m}$ ) and an increase of the half-life (to $\sim 1.8 \mathrm{~h}$ ).
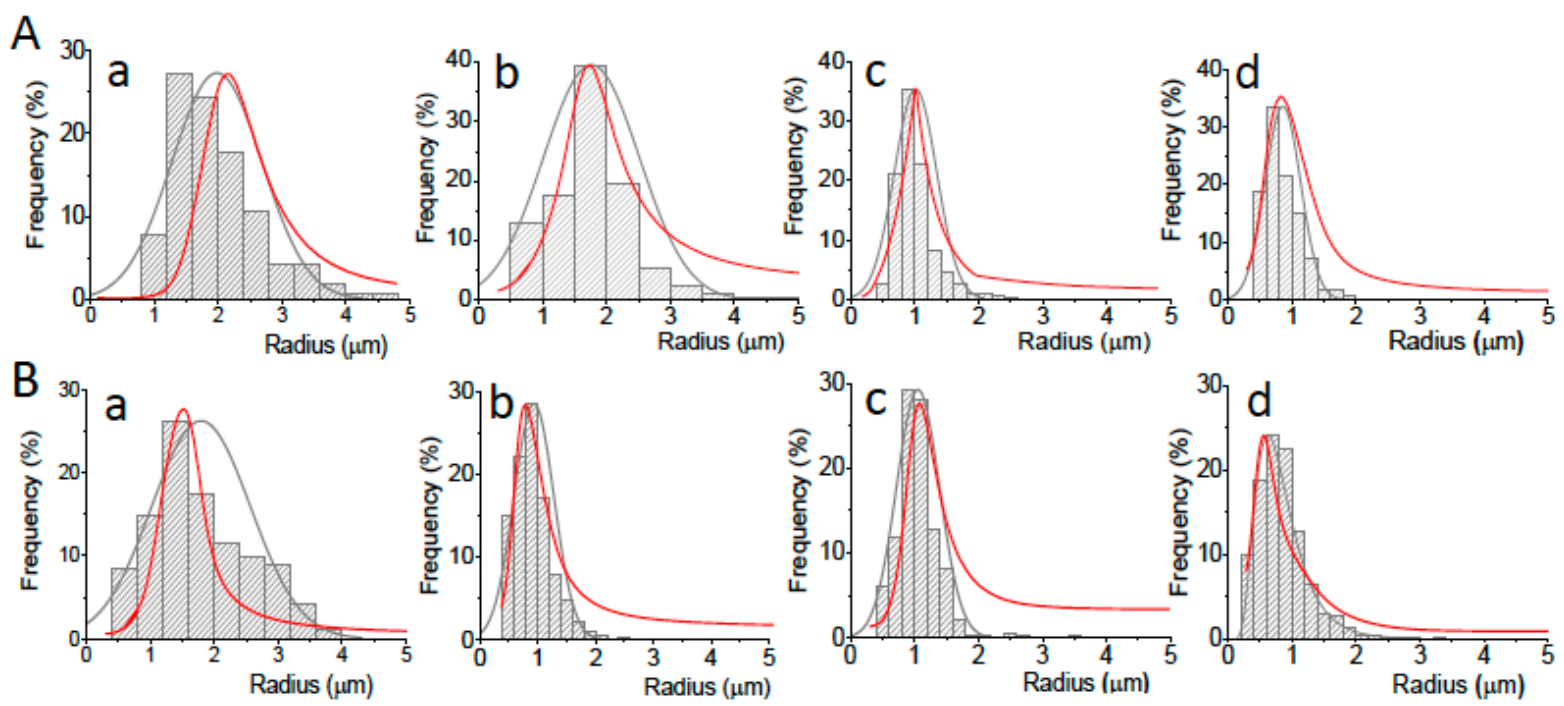

Figure 5. Size distributions of A) DMPC-shelled and B) DPPC-shelled microbubbles (red line: fit with a Gaussian function of the size histograms obtained from optical microscopy; black line: distributions obtained from the acoustical method). The MBs were prepared a) under $\mathrm{N}_{2}$; b) under $F$-hexane-saturated $\mathrm{N}_{2}$ phase; c) with $F$-hexane in the aqueous phase; and d) with $F$-hexane both present in the gaseous and aqueous phases. 

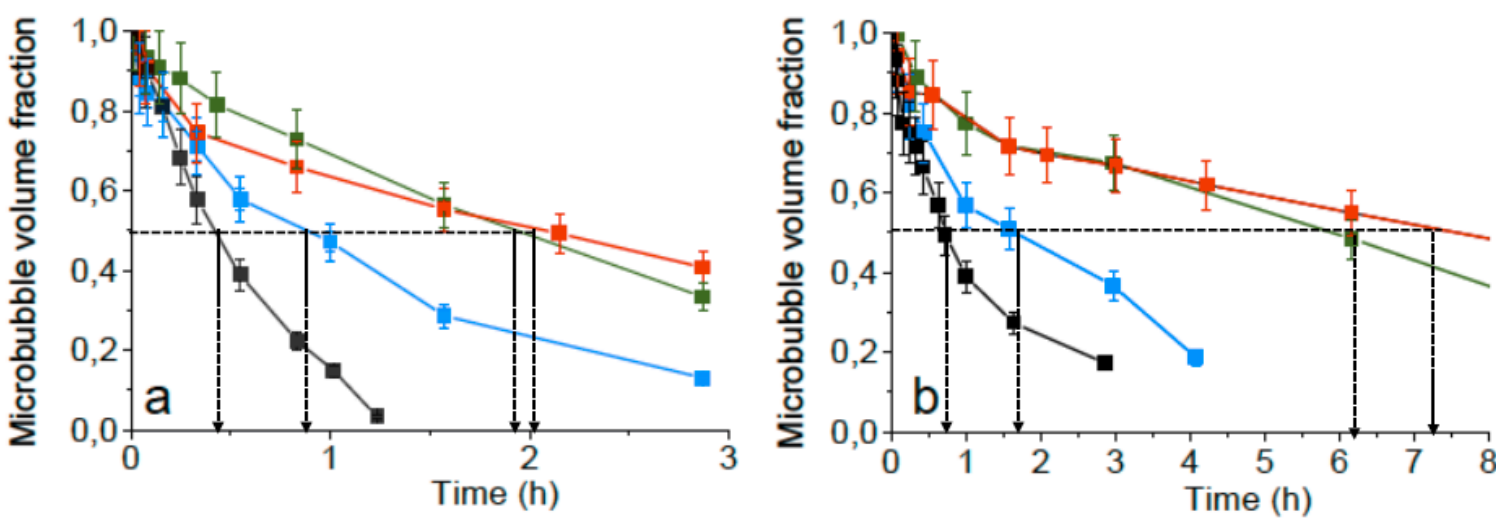

Figure 6. Time evolution of the volumes of a) DMPC and b) DPPC microbubbles prepared without $F$-hexane present (black); when $F$-hexane is introduced in the aqueous phase (blue), in the gas phase (green) and in both aqueous and gas phases (red). The arrows indicate the half-lives.

For DPPC, the mean radius of reference air microbubbles prepared without $F$-hexane is $\sim 2.0 \mu \mathrm{m}$ and their half-life is $\sim 0.8 \mathrm{~h}$. The presence of $F$-hexane, whether introduced in the aqueous or in the gas phase results in a significant decrease in bubble mean radius (to $\sim 1.0$ $\mu \mathrm{m})$ and in an increase of their half-life, the extent of this increase being more pronounced when the $F C$ is in the gas phase $(6.8 \mathrm{~h}$ vs. $1.7 \mathrm{~h})$. When $F$-hexane is introduced in both the gaseous and aqueous phases, one observes both a decrease in mean MB radius (to $<1.0$ $\mu \mathrm{m})$ and an increase in MB half-life (to $7.2 \mathrm{~h}$ ).

\section{Discussion}

We observed that the procedure of introduction of $F$-hexane, that is, either in the gas phase above, or in the aqueous phase below Langmuir monolayers, strongly impacts the compression isotherms of the latter. When the $F C$ is delivered in the gas phase, the LE to LC phase transition of the DPPC monolayer is shifted towards larger pressures but occurs at the same molecular area, while it is shifted towards larger areas at unchanged pressure when the $F C$ is provided via the aqueous phase. When introduced in the gas phase, $F$-hexane is rapidly incorporated in the phospholipid film but is also readily desorbed when the monolayer is compressed and finally completely expelled from the monolayers. On the contrary, when introduced in the aqueous phase, $F$-hexane remains trapped in the interfacial film. These 
unlike outcomes demonstrate that the phospholipid monolayer constitutes an effective barrier to the transfer of the fluorocarbon across the interfacial film.

Concerning the microbubbles, our results show that in all the cases investigated the FC has a beneficial effect on their characteristics, leading to smaller bubbles of highest stability. However, the extent of these benefits depends noticeably on the FC introduction procedure. When introduced in the aqueous phase, the $F C$ causes a small but significant decrease in MB mean radius, whilst when introduced in the gaseous phase, it strongly enhances their half-life, with a significant but less pronounced effect on their mean radius. Why is that so? It is quite remarkable that within each series investigated in this study all the phospholipidshelled microbubbles investigated in this study were found to reach a same mean radius of $\sim 1$ to $2 \mu \mathrm{m}$ with a similar narrow size distribution within a few minutes after preparation. After reaching this "critical" size the MBs of each four series were observed to persist for long periods of time (from $\sim 0.5$ to $7 \mathrm{~h}$ ) without any significant change in size. This is in agreement with several experimental investigations that reported that MBs having various initial sizes eventually stabilize around a uniform size range $(\sim 2-4 \mu \mathrm{m}$ in radius), provided they have a rigid lipid shell. ${ }^{33}$ For example, microbubbles subjected to acoustic pulsing were found to resist dissolution regardless of both initial diameter and shell composition. ${ }^{34}$ Reaching their critical size can cause small phospholipid-coated MBs $(\sim 1.5 \mu \mathrm{m})$ to have an order of magnitude longer half-lives than larger microbubbles $(\sim 5 \mu \mathrm{m})$ of similar shell composition. ${ }^{29}$ MBs (5 $\mu \mathrm{m}$ in diameter) produced in microfluidic channels were also seen to remain stable (for over $9 \mathrm{~h}$ ) at $1.5 \mu \mathrm{m} .{ }^{35}$ Microbubbles prepared by sonication typically have a mean diameter around 1-2 $\mu \mathrm{m}$, and those with larger initial size distributions shrink to this size range over time. ${ }^{36}$ This stabilization of MBs at a certain critical size is at odds with various mathematical models, such as that described by Epstein and Plesset, ${ }^{37-38}$ or more recent ones, ${ }^{39-41}$, which all predict that bubble dissolution is a catastrophic event during which the MBs are expected to disappear within minutes (or tens of minutes) once dissolution has begun. The process of dissolution of MBs coated with long-chain phospholipids was subsequently found to be non continuous, by contrast with what happens to bubbles coated 
with soluble surfactants, but to occur through discrete MB shell collapse events. Long-chain phospholipid-shelled MB collapse was shown to occur via the nucleation of microscopic folds, which leads to macroscopic folding events. ${ }^{33,39,42}$ This process, which results in cyclic wrinkle-to-smooth transitions of the microbubble shell can be modulated by adjusting the composition and microstructure of the lipid shell ${ }^{43}$ and strongly influences the rate of microbubble dissolution. ${ }^{44}$ It is reminiscent of the morphology transitions observed in lipid vesicles that are due in part to topological defects. ${ }^{31}$

We show that the fluorocarbon gas introduced either in the gas or the aqueous phase can help control the critical size reached by phospholipid-shelled microbubbles. This likely occurs by FC-fostered fluidization of the shell, as reflected, in particular, by the shift of the LE/LC transition to higher pressures observed in the isotherms of DPPC when $F$-hexane is present. This FC-driven fluidizing effect of phospholipid monolayers was previously reported for other volatile fluorocarbons. ${ }^{14-15}$ This supports the proposition that the FC hinders the formation of the micro-folds in the shell during bubble deflating. The consequences of the fluidizing effect of FCs on phospholipid monolayers have been discussed in view of developing lung surfactant substitutes, ${ }^{17}$ but have never been taken into consideration in the context of medical microbubbles. Understandably, the effect of the FC on critical bubble size is more pronounced with DPPC than with DMPC, since the latter is already in the fluid state at $25^{\circ} \mathrm{C}$. The smaller MB size reached when $F$-hexane is introduced in the aqueous phase $(1.0 \mu \mathrm{m}$ vs. $1.5 \mu \mathrm{m}$ when introduced in the gas phase) may be explained by the fact that the adsorption kinetics of the $F C$ to the interface is much slower, thus allowing more time for the MBs to deflate before the stabilizing effect of the FC can operate. The higher MB longevity observed when the $F C$ comes from the gas phase is logically related to its much higher concentration in the gas phase as compared to the aqueous phase.

\section{Conclusions and perspectives}

The mode of exposure to a fluorocarbon has a considerable impact on Langmuir and Gibbs monolayer behavior, and consequently on microbubble properties. We show that the 
phospholipid monolayer constitutes an efficacious barrier to the transfer of a fluorocarbon between the aqueous sub-phase and the supernatant gaseous phase. The FC gas can significantly decrease the critical size at which microbubbles stabilize. Its presence markedly increases their persistence. The extent of these effects depends substantially and unexpectedly on the fluorocarbon introduction mode. When introduced in the gas phase, the FC rapidly interacts with the phospholipid hydrophobic chains and hinders the collapse of the phospholipid shell, likely by fluidizing it. When introduced in the aqueous phase, the diffusion of the $F C$ to the interface is considerably slower, which allows reaching smaller critical MB sizes. The highest microbubble stability is achieved when the FC is introduced via the gas phase. These results support the view that fluorocarbons, which are frequently used in the formulation of medical microbubbles, and are often considered as simple osmotic agents, definitely play a more complex role by interacting with the phospholipid monolayer and modifying its behavior. Moreover, this interaction in a non-equilibrium system, depends strongly on the experimental protocol of introduction of the FC. All this suggests the possibility of tailoring the fluorocarbon structure in order to better control its interactions with the phospholipid shell components towards the preparation of smaller and more stable microbubbles.

\section{References}

(1) Schutt, E. S.; Klein, D. H.; Mattrey, R. M.; Riess, J. G. Injectable microbubbles as contrast agents for diagnostic ultrasound imaging: The key role of perfluorochemicals. Angew. Chem. Int. Ed. 2003, 42, 3218-3235.

(2) Riess, J. G. Fluorocarbon-based injectable gaseous microbubbles for diagnosis and therapy. Curr. Opin. Colloid Interf. Sci. 2003, 8, 259-266.

(3) Lindner, J. R. Microbubbles in medical imaging: Current applications and future directions. Nat. Rev. Drug Disc. 2004, 3, 527-532.

(4) Unger, E. C.; Porter, T.; Culp, W.; Labell, R.; Matsunaga, T.; Zutshi, R. Therapeutic applications of lipid-coated microbubbles. Adv. Drug Deliv. Rev. 2004, 56, 1291-1314.

(5) Hernot, S.; Klibanov, A. L. Microbubbles in ultrasound-triggered drug and gene delivery. Adv. Drug Deliv. Rev. 2008, 60, 1153-1166. 
(6) Ferrara, K. W.; Borden, M. A.; Zhang, H. Lipid-shelled vehicles: Engineering for ultrasound molecular imaging and drug delivery. Acc. Chem. Res. 2009, 42, 881-892.

(7) Peyman, S. A.; Abou-Saleh, R. H.; Evans, S. D. Microbubbles for therapeutic delivery. Ther. Deliv. 2013, 4, 539-542.

(8) Aryal, M.; Arvanitis, C. D.; Alexander, P. M.; McDannold, N. Ultrasound-mediated bloodbarrier disruption for targeted drug delivery in the central nervous system. Adv. Drug Deliv. Rev. 2014, 72, 94-109.

(9) Sirsi, S. R.; Borden, M. A. State-of-the-art materials for ultrasound-triggered drug delivery. Adv. Drug Deliv. Rev. 2014, 72, 2-14.

(10) Owen, J.; Crake, C.; Lee, J. Y.; Carugo, D.; Beguin, E.; Khrapitchev, A. A.; Browning, R. J.; Sibson, N.; Stride, E. A versatile method for the preparation of particle-loaded microbubbles for multimodality imaging and targeted drug delivery. Drug Deliv. Transl. Res. 2018, 8, 342-356.

(11) Chong, W. K.; Papadopoulou, V.; Dayton, P. A. Imaging with ultrasound contrast agents: current status and future. Abdom. Radiol. 2018, 43, 762-772.

(12) Mehta, K. S.; Lee, J. J.; Taha, A. A.; Avgerinos, E.; Chaer, R. A. Vascular applications of contrast-enhanced ultrasound imaging. J. Vasc. Surg. 2017, 66, 267-273.

(13) Riess, J. G. Injectable oxygen carriers (blood substitutes) - Raison d'être, chemistry, and some physiology. Chem. Rev. 2001, 101, 2797-2920.

(14) Gerber, F.; Krafft, M. P.; Vandamme, T. F.; Goldmann, M.; Fontaine, P. Preventing crystallization of phospholipids in monolayers: a new approach to lung surfactant therapy. Angew. Chem. Int. Ed. 2005, 44, 2749-2752.

(15) Gerber, F.; Krafft, M. P.; Vandamme, T. F.; Goldmann, M.; Fontaine, P. Fluidization of a dipalmitoylphosphatidylcholine monolayer by fluorocarbon gases: potential use in lung surfactant therapy. Biophys. J. 2006, 90, 3184-3192.

(16) Sirsi, S.; Pae, C.; Oh, D. K. T.; Blomback, H.; Koubaa, A.; Papahadjopoulos-Sternberg, B.; Borden, M. Lung surfactant microbubbles. Soft Matter 2009, 5, 4835-4842.

(17) Krafft, M. P. Overcoming inactivation of the lung surfactant by serum proteins: a potential role for fluorocarbons? Soft Matter 2015, 11, 5982-5994.

(18) Kabalnov, A.; Klein, D.; Pelura, T.; Schutt, E.; Weers, J. Dissolution of multicomponent microbubbles in the blood stream: 1. Theory. Ultrasound Med. Biol. 1998, 24, 739-749.

(19) Kabalnov, A.; Bradley, J.; Flaim, S.; Klein, D.; Pelura, T.; Peters, B.; Otto, S.; Reynolds, J.; Schutt, E.; Weers, J. Dissolution of multicomponent microbubbles in the blood stream: 2. Experiment. Ultrasound Med. Biol. 1998, 24, 751-760.

(20) Szijjarto, C.; Rossi, S.; Waton, G.; Krafft, M. P. Effects of perfluorocarbon gases on the size and stability characteristics of phospholipid-coated microbubbles - Osmotic effect versus interfacial film stabilization. Langmuir 2012, 28, 1182-1189. 
(21) Nguyen, P. N.; Trinh Dang, T. T.; Waton, G.; Vandamme, T.; Krafft, M. P. A nonpolar, nonamphiphilic molecule can accelerate adsorption of phospholipids and lower their surface tension at the air/water interface ChemPhysChem 2011, 12, 2646-2652.

(22) Lehmler, H.; Jay, M.; Bummer, P. Mixing of partially fluorinated carboxylic acids and their hydrogenated analogues with dipalmitoylphosphatidylcholine at the air-water interface. Langmuir 2000, 16, 10161-10166.

(23) Matyszewska, D.; Tappura, K.; Ora, G.; Bilewicz, R. Influence of perfluorinated compounds on the properties of model lipid membranes. J. Phys. Chem. B 2007, 11, 99089918.

(24) Nakahara, H.; Lee, S.; Krafft, M. P.; Shibata, O. Fluorocarbon-hybrid pulmonary surfactants for replacement therapy - A Langmuir monolayer study. Langmuir 2010, 26, 18256-18265.

(25) Nakahara, H.; Krafft, M. P.; Shibata, A.; Shibata, O. Interaction of a partially fluorinated alcohol $(\mathrm{F} 8 \mathrm{H} 11 \mathrm{OH})$ with biomembrane constituents in two-component monolayers. Soft Matter 2011, 7, 7325-7333.

(26) Abou-Saleh, R. H.; Peyman, S. A.; Johnson, B. R.; Marston, G.; Ingram, N.; Bushby, R.; Coletta, P. L.; Markham, A. F.; Evans, S. D. The influence of intercalating perfluorohexane into lipid shells on nano and microbubble stability. Soft Matter 2016, 12, 7223-30.

(27) Klibanov, A. L. Preparation of targeted microbubbles: ultrasound contrast agents for molecular imaging. Med. Biol. Eng. Comput. 2009, 47, 875-882.

(28) Nguyen, P. N.; Veschgini, M.; Tanaka, M.; Waton, G.; Vandamme, T.; Krafft, M. P. Counteracting the inhibitory effect of proteins towards lung surfactant substitutes: a fluorocarbon gas helps displace albumin at the air/water interface. Chem. Commun. 2014, 50, 11576-11579.

(29) Rossi, S.; Waton, G.; Krafft, M. P. Small phospholipid-coated gas bubbles can last longer than larger ones. ChemPhysChem 2008, 9, 1982-1985.

(30) Rossi, S.; Waton, G.; Krafft, M. P. Phospholipid-coated gas bubble engineering - Key parameters for size and stability control as determined by an acoustic method. Langmuir 2010, 26, 1649-1655.

(31) Hirst, L. S.; Ossowski, A.; Fraser, M.; Geng, J.; Selinger, J. V.; Selinger, R. L. B. Morphology transition in lipid vesicles due to in-plane order and topological defects. Proc. Nat. Acad. Sci. USA 2013, 110, 3242-3247.

(32) Mitev, D. J.; Ivanova, T.; Vassilieff, C. S. Kinetics of lipid layer formation at interfaces. Coll. Surfaces B: Biointerfaces 2002, 24, 185-192.

(33) Kwan, J. J.; Borden, M. A. Lipid monolayer collapse and microbubble stability. Adv. Coll. Interface Sci. 2012, 183-184, 82-99. 
(34) Borden, M. A.; Kruse, D. E.; Caskey, C. F.; Zhao, S.; Dayton, P. A.; Ferrara, K. W. Influence of lipid shell physicochemical properties on ultrasound-induced microbubble destruction. IEEE Trans. Ultrason.Ferroelectr. Freq. Control 2005, 52, 1992-2002.

(35) Talu, E.; Hettiarachchi, K.; Powell, R. L.; Lee, A. P.; Dayton, P. A.; Longo, M. L. Maintaining monodispersity in a microbubble population formed by flow-focusing. Langmuir 2008, 24, 1745-1749.

(36) Feshitan, J. A.; Chen, C. C.; Kwan, J. J.; Borden, M. A. Microbubble size isolation by differential centrifugation. J. Colloid Interf. Sci. 2009, 329, 316-324.

(37) Epstein, P. S.; Plesset, M. S. On the stability of gas bubbles in liquid-gas solutions. J. Chem. Phys. 1950, 18, 1505- 1509.

(38) Duncan, P. B.; Needham, D. Test of the Epstein-Plesset model for gas microparticle dissolution in aqueous media: Effect of surface tension and gas undersaturation in solution. Langmuir 2004, 20, 2567-2578.

(39) Borden, M.; Longo, M. Dissolution behavior of lipid monolayer-coated, air-filled microbubbles: Effect of lipid hydrophobic chain length. Langmuir 2002, 18, 9225-9233.

(40) Sarkar, K.; Katiyar, A.; Jain, P. Growth and dissolution of an encapsulated contrast microbubble: effects of encapsulation permeability. Ultrasound Med. Biol. 2009, 35, 13851396.

(41) Katiyar, A.; Sarkar, K.; Jain, P. Effects of encapsulation elasticity on the stability of an encapsulated microbubble. J. Colloid Interf. Sci. 2009, 336, 519-525.

(42) Kim, D. H.; Costello, M. J.; Duncan, P. B.; Needham, D. Mechanical properties and microstructure of polycrystalline phospholipid monolayer shells: novel solid microparticles. Langmuir 2003, 19, 8455-8466.

(43) Pu, G.; Borden, M. A.; Longo, M. L. Collapse and shedding transitions in binary lipid monolayers coating microbubbles. Langmuir 2006, 22, 2993-2999.

(44) Lozano, M. M.; Longo, M. L. Complex formation and other phase transformations mapped in saturated phosphatidylcholine/DSPE-PEG2000 monolayers. Soft Matter 2009, 5, 1822-1834. 
Table of Content

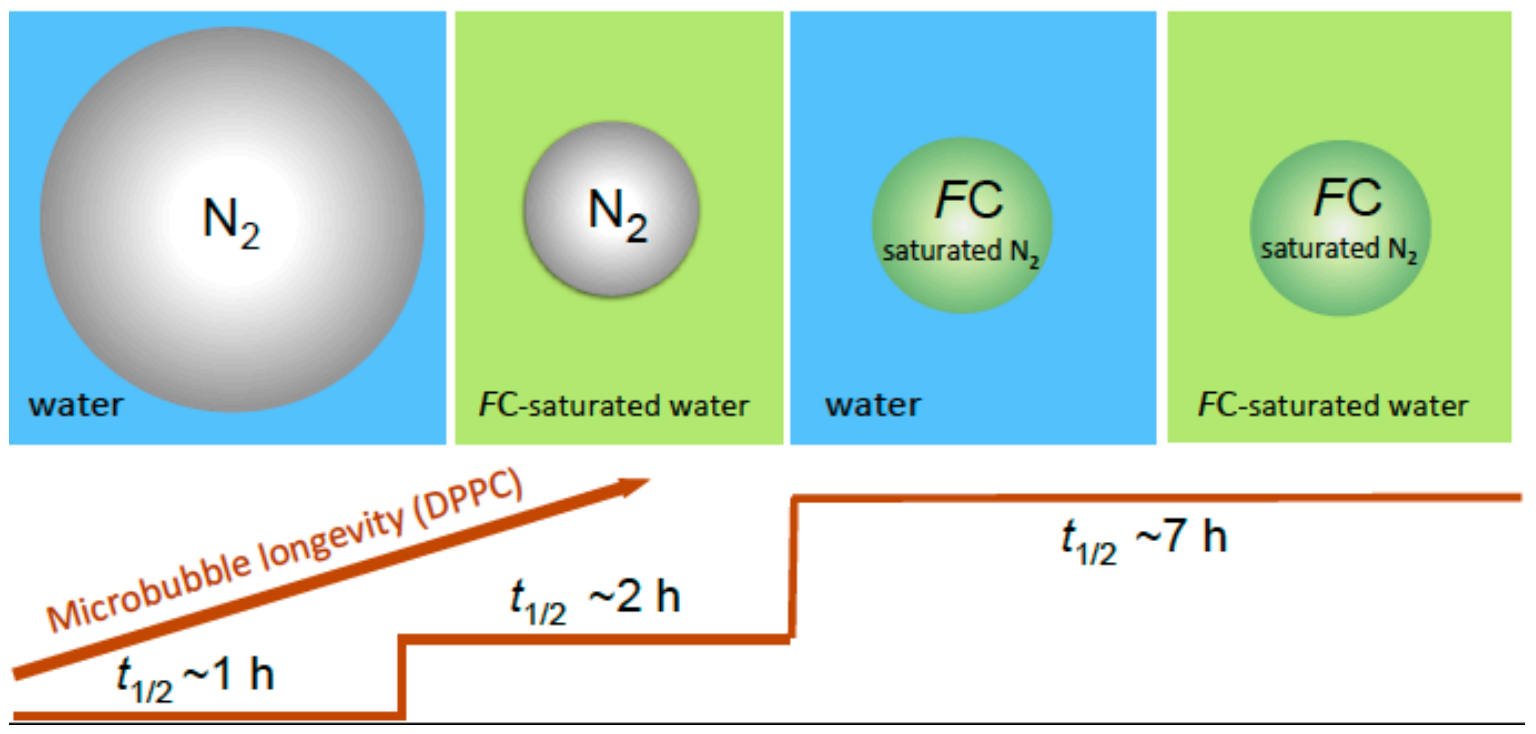

